\title{
The Effect of Optical Formation on Highlighting the Features of Urban Identity
}

\author{
D.O.I - 10.51201/Jusst12618
}

http://doi.org/10.51201/Jusst12618

\author{
Ahraou wafa $^{1}$ HyndaBoutabba $^{2}$ Abdallah FARHI $^{3}$ \\ 1.phD student, department of architecture Biskra University, Algeria. \\ 2.Professor, GTU institute, M'sila University, Algeria. \\ 3. Professor, department of architecture, Biskra University, Algeria.
}

\begin{abstract}
The study includes four chapters, the first is devoted to reviewing the research problem, its importance, objectives and limitations in addition to defining terms.

The second chapter contains the theoretical introduction that includes a definition of optical art and its impact on highlighting features of urbanidentity.

The third chapter examines several models in optical formation, where several architectural designs were discussed by its role in highlighting the features of urbanidentity.

The fourth chapter includes the results and observations of the role of art and opticalformation in highlighting the urbanidentity. This is because it adds an aesthetic touch and an expression of various creative and unfamiliar ideas to the designer in a waythat occurs a fundamental change in the fixed rules of the viewer and his/her expectations for designs where the creative touch is in the overlay, communication, separation, penetration, segmentation of shapes and colors, and selection of materials needed to implement these designs have the biggest role in highlighting the features of urbanidentity.

Where we found that the optical formation of its various types is the main component of architecture that it boasts of, and with what it contains creative ideas that express the reality of urbanidentity.
\end{abstract}

\section{Research Problem and the Need for it:}

Architecture was and still the primary facade of culture, economy and civilization of nations, and we are in the timeof the enormous development of knowledge and technology that is an integral 
part of architecture.It reflects in a competitive way what countries have come to with development in thought, civilization and technology, and because architecture was one of the most important criteria in classifying the progress and development of countries, so we need to advance ideas by stimulating modern ideas and support them to raise the level of awareness of society to reflect on the role of architectural design and optical formation in highlighting landmarks of urban identity. To achieve this, studies in the evolution of architectural building design should be undertaken to study complete and analytical study in both artistic and architectural sides, as art and optical formation are of great importance in highlighting landmarks of urban identity, because the introduction of art and optical formation in architecture is the first step towards an urbanismrenaissance in countries and thus an economic renaissance, because architecture is the backbone and facade of the economy, and through this technical and architectural studies, we can determine the positives of previous architectural experiences and avoid negatives, and to employ art in the ideal way to display the culture and heritage of civilized countries and urban identity through its integration with architectural designs, and the optical artistic formation makes the recipient and the researcher curious about the reason behind design: was art used for a functional, historical, or aesthetic aspect, and what role or purpose is behind these designs that the designer wants to show in architecture and make the beholder think about the impact of art and optical formation to highlight the features of urban identity, and how to benefit from it in future designs.

\section{Research Aims:}

The aim of it was presented in the previous paragraph, and it is summarized in: the effect of optical formation on highlighting the features of urban identity.

\section{Research Limits:}

Study applications of optical formation.

An analytical study of several buildings that used optical formation in their design.

Optical Arts are the oldest artistic activity through which man produced pictures and figures that are visible, so we can call it optical arts, which is the art that is concerned with producing spatial artworks that take up a place, and we will deal with art and opticalformation in highlighting the urbanidentity.

We have found from the above that architecture is one of the most important types of art that expresses the history of its design and is characterized by its pure nature, including economic, cultural and civilizedchanges, in addition to its material, visual reality that man coexists with him and is influenced by him at all times and through all activities. Architecture is functional art that 
is defined by the place in which we live and which creates a plastic framework that surrounds our life.

The architect and architecture have a great role in shaping urban reality and shaping different environments and influencing individuals as he/she designs and shapes architecture, then it influences us, as Winston Churchill said: "We make our buildings, and then they make us and organize our life."

Modern architecture first appeared in the first half of the twentieth century and the one who has the favor in the development in the art of architecture and its integration with optical art is the artist Victor Vasarely, who created the optical art that is based on the visual perception technique of similar shapes and floors and relied on designs that inspire the recipient and this art is called by the term op art which is an abbreviation of optical in the sense of visual and art in the sense of art, and there are other artists such as C.rostow and Bridget Riley who have made important additions to architectural optical art.

The large and accelerated development of the completed architecture reflects the development of real life and highlights its urbanidentity, and therefore we conclude the importance of art and optical formation in its various applications and creative fields in demonstrating urban identity and developing thoughtful expression of the mixture of living reality, mental desire, and what we see from designers 'ideas as the biggest contributor to crafting the thought-provoking visual achievement and expressing the urban identity.

As the mind distinguishes between two configurations in architecture, they are the stage of the sanity of architecture and the stage of visualizing architecture, and we all agree in the stage of rationalization of architectural achievements, but we differ in the stage of perception, which is an area for diligence, creativity and the area of human mobility.

From the above, we must ask the following questions:

What are the types of architectural optical illusion and formation?

How did these formations highlight the features of urban identity?

What is the role of these visual formations in architectural designs was their role only aesthetic or was it a service role too?

In this chapter, we will deal with the answer to the first question, and we will deal with the rest of the questions in the third chapter.

There are two types of architectural optical illusion and formation: 
- External: means the exterior design and the general shape of the building, where the design of the building has three main aspects which are the need or function, shape and environment

We will talk about some types of optical formations used.

- Openings: one of the most important elements

Offormation and its expression varies in different

Ways with the difference of the building and

The entrance is the most important architectural

Element in the formation where with it,

We can specify the levels of the interface elements.

- Motifs: it has the largest role in the opposite

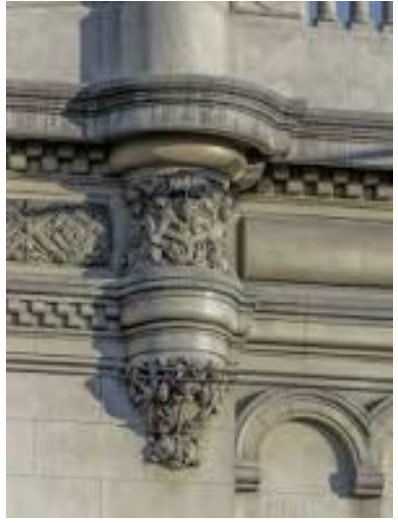

Figure (1)

Ofcivilized culture and legacy is the most important

Element of formation that gives the

Building its urban identity.

- Building mass: may consist of an

Infinitenumber of shapes, it may be

Linear solo or component of a parallel

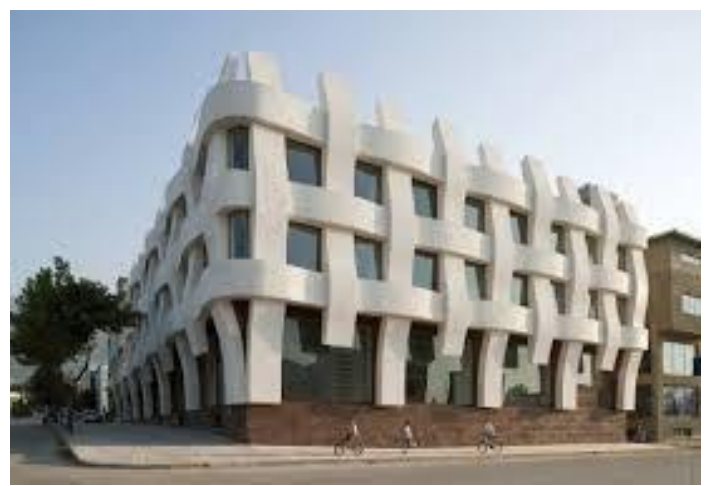

Set of blocksororthogonal blocks and construction design

Figure (2)

May be streamlined or italic.

-Internal: It is the most important and diversified foroptical formation. Formation and optical illusion hasbegun in the Renaissance in designing of large palaces and halls, where large murals containing drawings and pictures of owners of palaces and kings were created of a nature consistent with culture and civilized heritage that give architecture a historical and social identity.

In the 1960s, the concept of Psychological Architecture emerged. It expresses the cooperation between psychologists and architects in employing building design to fulfill the psychological needs of individuals.

The subscription area that you combine

Architects and psychologists that both from 
Sciences that deal with human behavior

In a direct way where psychology was

theorifical science and architecture is

applied science. The internal optical

formation helps to change the rules of

visual perception of the spaces for the

viewer where it gives a feeling of zooming

in or out visually or in depth or narrow depending

on a mixture of scientific theories and modern art.

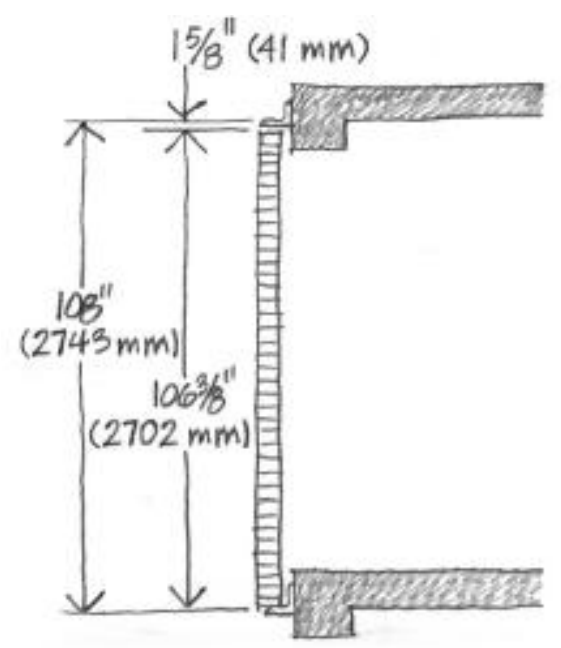

Figure (3)

Optical formation in internal architectural

Design depends on:

-Brightness and contrast: depends on the

variety oflighting, lengths and heights

(Figure 3) and contrast.

It is a rapid transition from state

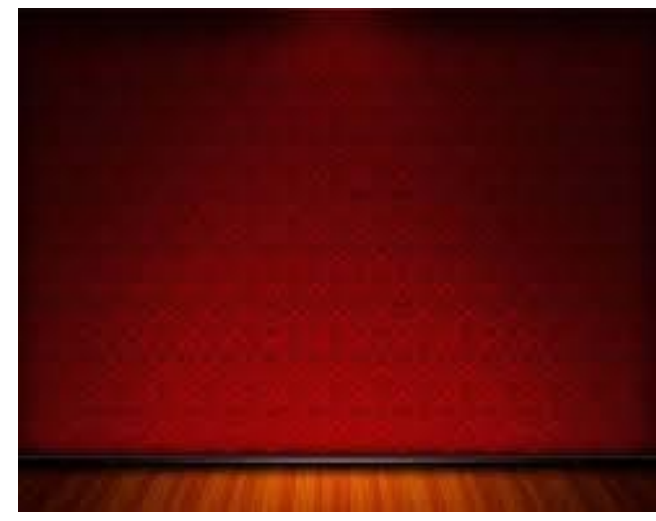

Figure (4)

to state. Joan Meyers Levy made the markby studying the relationship between the height of the void roof and thinking style, she concluded that the rooms with good heightmade people faster to configure words from random and special letters. Words that suggest restriction and confinement and vice versa, but for very high ceilings, people showed speed in words concerning freedom, she encouraged people to think in a holistic, abstract manner without focusing on parts and details, and here we find how this difference in height emerged in which an urban identity of the roof affected human behavior.

- Color: It is the most important optical element related to light, and these two elements are combined the most elements affecting cognition.

Together with 600 people, including university students, the psychologists in British Columbia have done experiments to study the effect of wall color on the visualization process." Figure 4"

Where basic behavioral cognitive experiments were carried out within different colored walls, and the differences were significant. For example, the color red as the color of danger showed 
students more skill for choices that require focus, accuracy, and attention to detail, such as memorizing a set of numbers.

Scientists have linked the meaning of color

and behavior, and here we find how it arose

the color by giving the wall an urban

identity that affected the behavior. We will

cover several experiments in the third chapter.

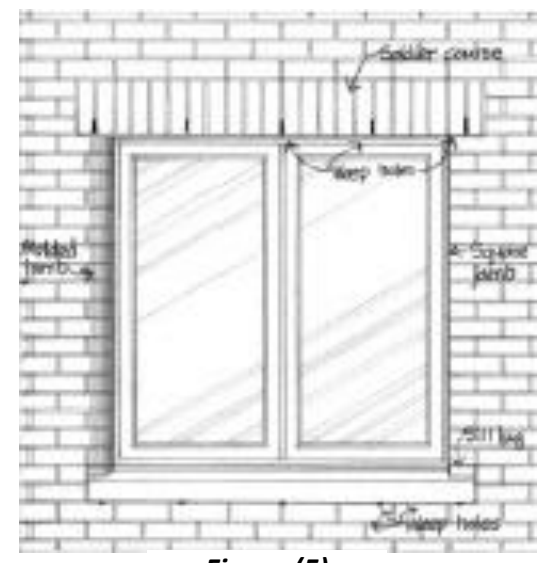

Figure (5)

-Various combinations: decorating the walls with panels or floors through horizontal, vertical, diagonal and dashed lines, or diversification in the furniture, windows and perspective. We will look at some examples in chapter three.

"Figure 5"

In this chapter, we will cover examples of external optical formation and how this formation contributed to highlighting landmarks of architectural identity.

We will talk first about Liv hospital in Istanbul-Turkey of Global Medical Care Company, we will discuss its internal and external optical design and composition.

First: External Optical Formation:(Note in Figure 6)

The first thing that catches the

Eyeis the gate with the

streamlined shapecomposed

of glass panels thatgive aesthetic

and eye comfortat the

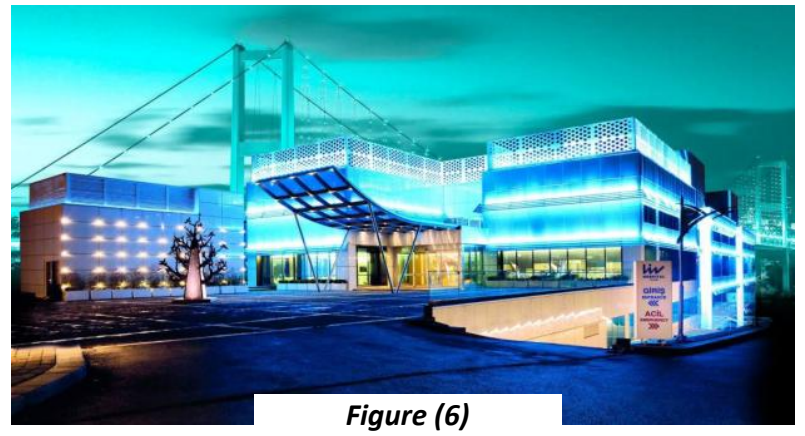

reception of the architectural facade then we note facade walls different in formation. Most of the walls are decorated with blue lighting that gives a kind of mental relaxation, and this is one of the resultsof the experiments whichpsychologists have done in British Columbia University which their principle is mentioned in chapter two, then we note the emergency entrance, despite its presence at the front of the hospital, but it is easy to enter the hospital far from the crowd of the main entrance, whose walls are decorated with a mixture of glass and blue colors. All these combinations that were mentioned contributed to the functional aspect of the building in terms of 
ease of movement, organization, aesthetic and psychological aspects which the combination of these formations helped to add aesthetic to the design of the building alsolights and the flow of the gate play a role in influencing the psyche of individuals, which increases relaxation to move away from a condition of stress for most individuals when they enter hospitals.

From the above, we find that the external optical formation suggested to us that the building provides a service aspect that aims to improve the psychological state of individuals.

That is, these external formations, contributed to highlighting the characteristics of the urbanidentity of the building, and it is a medical service identity.

Second: Internal Optical Formation:

We notice from figures (7) and (8)

many optical formations, and we will

start talking about the ceiling height

and visual effects in it, we note that

the ceilings are high which gives

feeling of comfort, freedom and lack of

detention for individuals and

lights were distributed consistently

with the dimensions of the halls and

hallways that give the feeling of
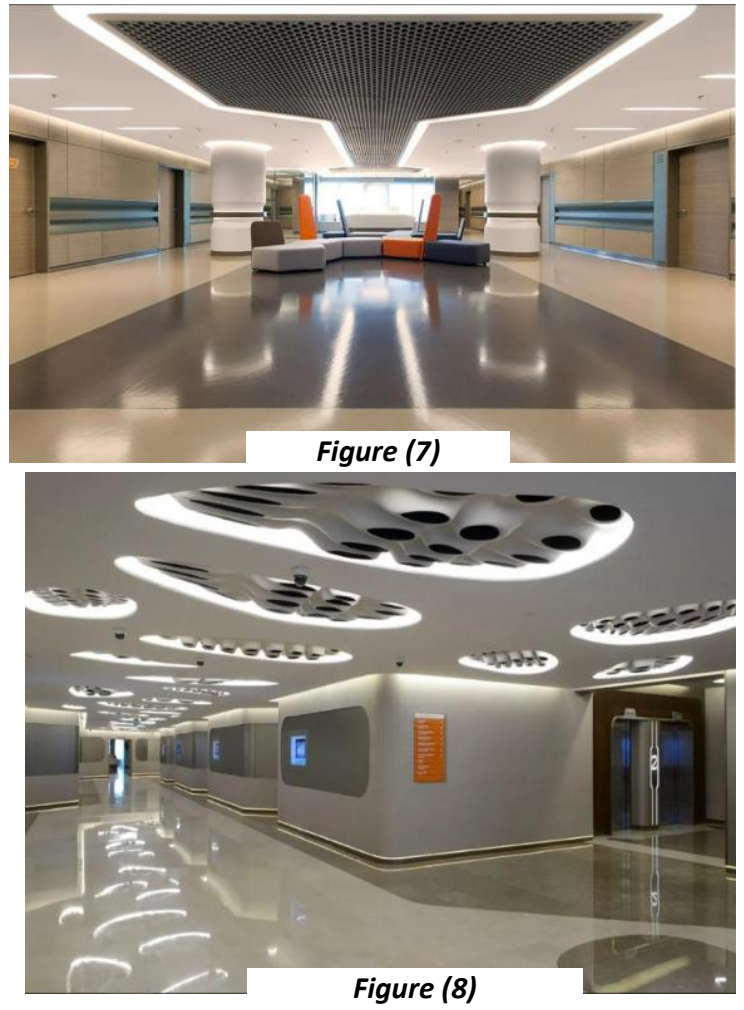

distress which one is felt in deep buildings, which natural light does not reach its rooms and halls, and we note that the ceilings are decorated with a mixture of geometric shapesto give the kinetic character to break the feeling of boredom and turns the mind away, through meditation, from stresswithin the hospital, as well the walls have been diversified in its color and lighting and adding service accessories in a coordinated way, it distances the feeling of inertia.

In Figures (7), (8) and (9), we note the diversification in the floors where visual diversification was used in every part to suit it.

Different variable depth and geometrical shapes that suggest calm in waiting halls have been used, and the floors of horizontallines in corridors and lobbies inspired stability and constancy.

Likewise, the columns in which 
the formation was used were lost

because they are designed in

different shapes to suit colors and

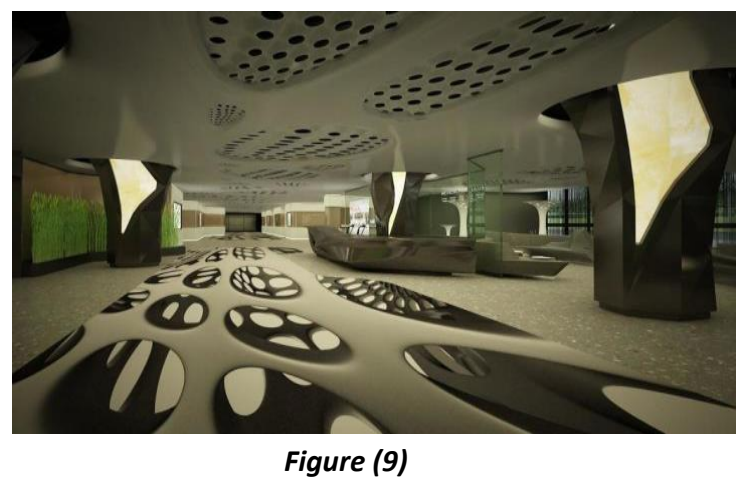

ceiling and floorlighting. Some are designed in a variable width and high way in addition to lighting consistency of columns with furniture.

From the previously mentioned internaloptical formations, we find that all of these formations were achieving the psychological and service goal of the building, these formations have highlighted the features of the urbanidentity of the building, namely the medical and service identity.

Finally, we find that the internal and external optical formations that were used are the main element which reflects the service and technical goal, linking them together and highlighting them in urban design.

We will now talk about the Monastero Santa Rosa Hotel in Conca Dei Marini-Italy and take up external and internal opticalformations in it.

First: External Optical Formation:

We must talk about the hotel's

distinctive andunique location,

which, in itself, is anoptical

formation different from

the rest of the hotels in the world

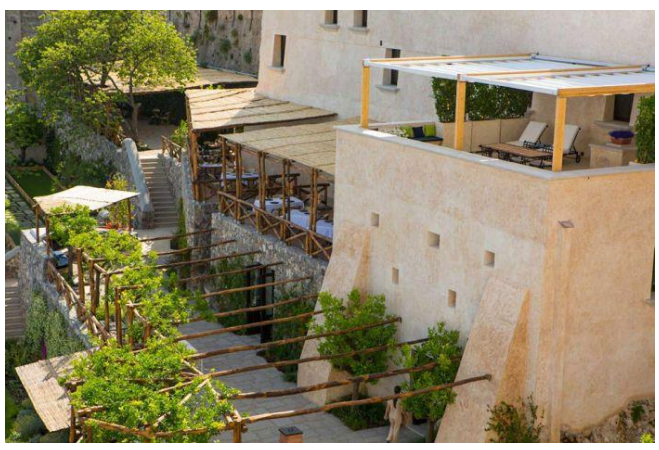

Figure (10)

where this hotel is designed on slope

on the Gulf of Salerno, this hotel wasand still retains its style where we find the original forms as in figures (10), (11) and (12) that the external design and the covering of the facade mix with the surrounding nature also retaining its historical identity, as we note that the facade walls do not contain any additions or colors, but rather retain simplicity adhering to its historical style, and we 
find that the building is inseparablefrom the surrounding environment by connecting facades with nature by logs of wood

and trees distributed on the sides of the hotel. This gives the hotel

a wonderful viewand unprecedented harmony for a hotel with nature, these harmony, simplicity and beauty of the hotel also the scenic locationgives

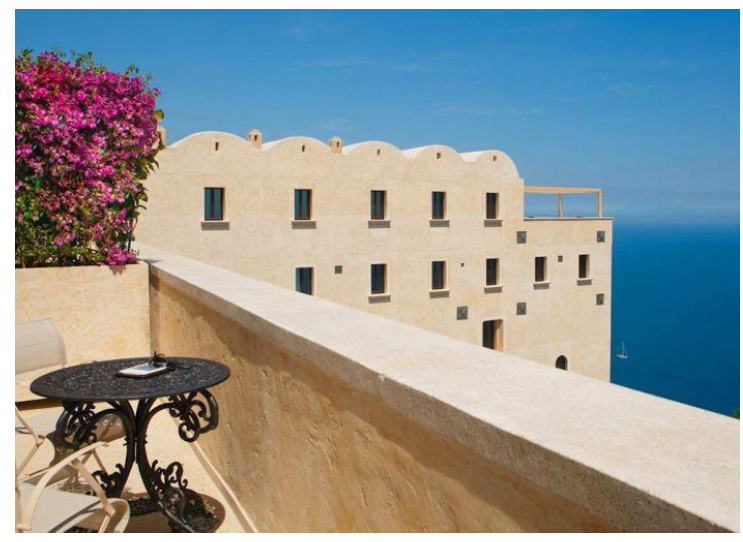

Figure (11)

desire to people to visitit because of the comfort for the soul by meditating with this beauty. We find that all these formations are simple and harmonious aim to attract visitors where we find itbrought out the tourist identity of the building with maintaining its historical identity.

Second: Internal Optical Formation:

We will first talk about the rooms where we find them in figure (13) and (14) that its general nature indicates it is characterized by simplicity and avoid evaosaration in formation where we find that the walls and ceilings are of a fixed whit stability and

eye comfort due to the enormous

optical formation for nature outside also, art muralpaintings were added

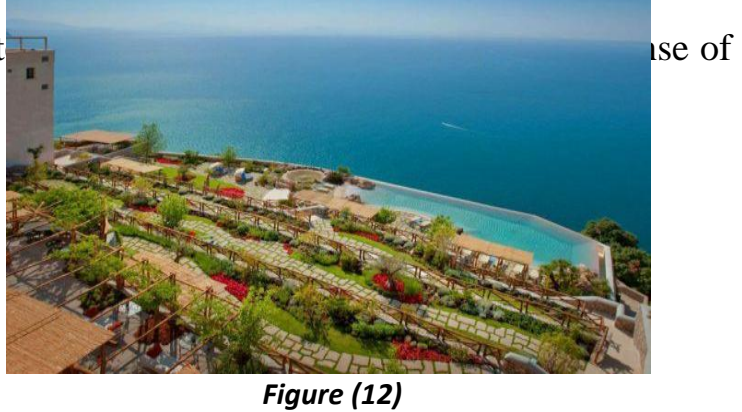

for a classy aesthetic touch, but for lighting, distributed lights were put to enlighten the entire rooms with a fixed color view for the construction is adopting on the light of natural

light which is comfortable for the

eye, and gives visitors

psychological and mental relief, also the total dependence in interior opticalformationwas in the furniture, where we find the

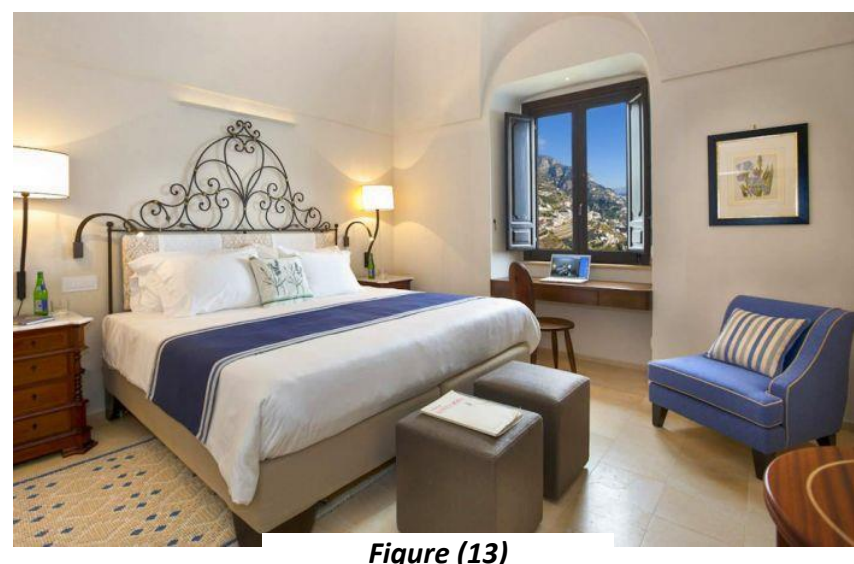

Figure (13) 
mixture between modern furniture and

old historical furniture and their harmony

together to give the rooms a luxurious

aesthetic touch away from overstated of

optical formation, we find that all optical

formationsused gives a feeling of comfort

and desire of visitors to stay within

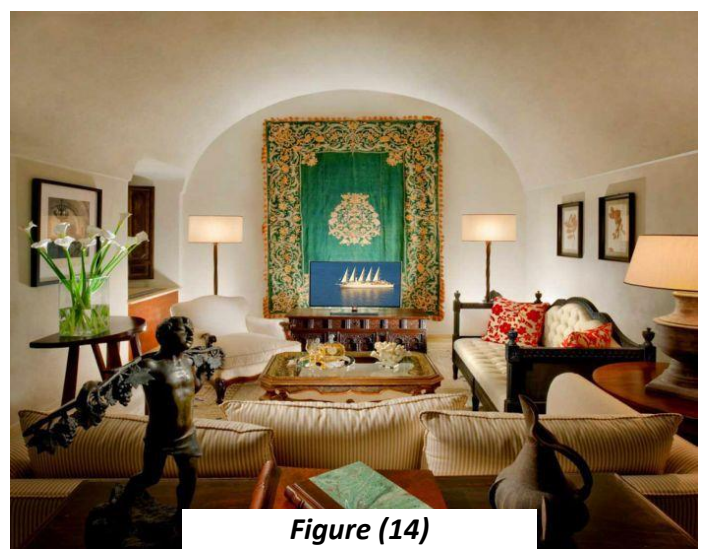

this mixture of civilization, nature and history.

In figures (15) and (16) we find that

the design of the dining hall and

bathrooms are no less creative than the

rest of this hotel, where we notice that

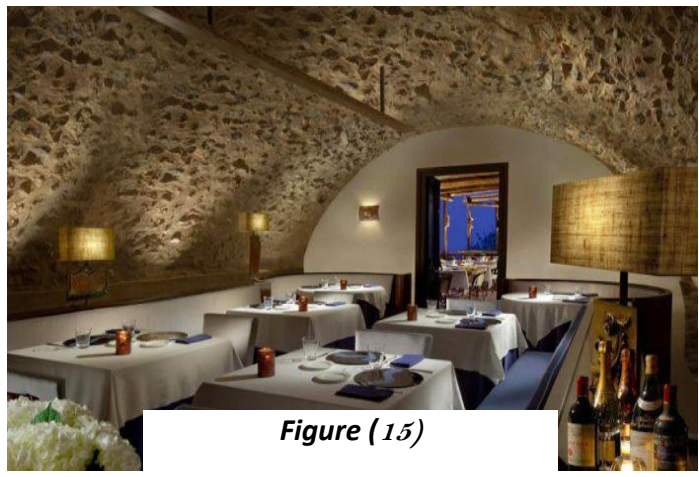

some of its ceilings and walls have notchanged its historical identityand lighting in food hall distributed harmoniously, that shows the historical identity of ceiling andfurniture of about the same colors increased the aesthetic of the hall and the feelingof comfort. As for the bathrooms, some historical walls have been preserved, relying on lighting to highlight aesthetics, plus luxurious design for lighting floors and ceilings that reflects the history of this hotel as well we find that the internal visualformations for the bathrooms and the dining hall did not give up its historical identity in its design and launched from this identity to add

to the building an aestheticand touristidentity.

We find from all these internal

and externalformations that it

did a functional work served the

hotel to attract tourists and aesthetics

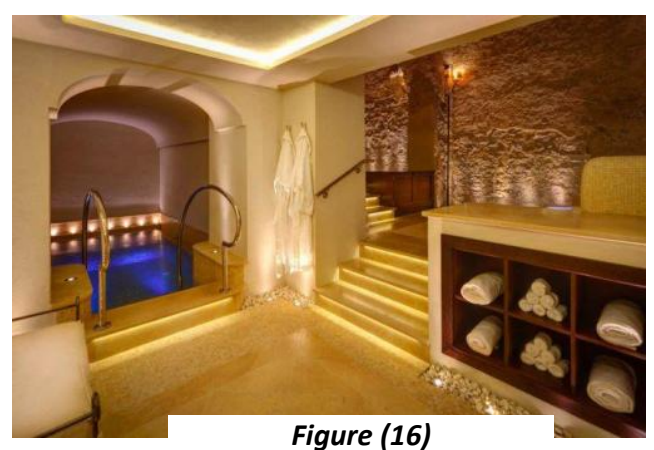

that highlights the historical andtouristidentityfor the hotel.

We will now talk about the Agora Lelystad Theater in the Netherlands, which is an architectural icon and masterpiece, and what has been used of internal and external visual formations in the theater's design. 
First: External Optical Formations:

In Figures (17) and (18), we find that the facade of the building is composed of several geometric shapes showing a distinctive artistic painting in addition to a wide use of glass in the facade,

which made the mixture between

it and the geometric harmonious

shapes in an unfamiliar, attractive way.

The building appears in an orange color,

which is an unusual color for buildings

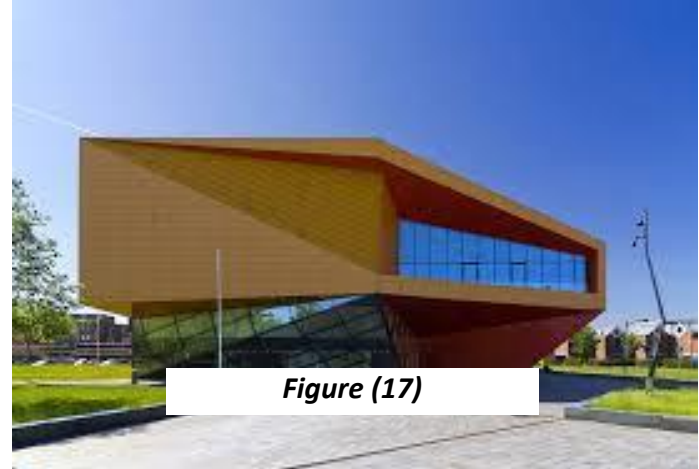

that increases the attractiveness of its design, and nignt lıghtıng appears

through the glass facade, making the building appear as glowing; the thing that makes this building a remarkable and interesting architectural building and evokes the desire and curiosity of the viewer in

exploring this building from the

inside. We also find that its

designand external formations

drew attention to the building

from the viewers and highlighted

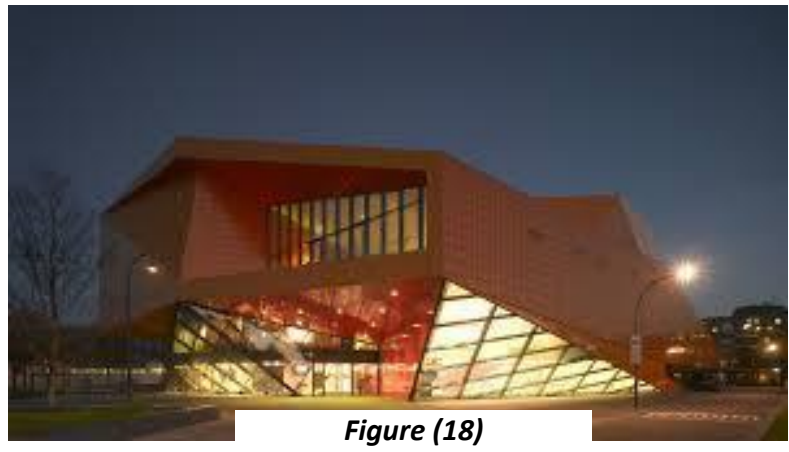

a large aesthetic and artistic identity for the theater. These formations reflected the artistic function of the theater.

Second: Internal Optical Formations:

From figures (19) and (20) and (21)

we find that the main reliance in

the internal visual formation was

on thecolors that were carefully

chosen, and these colors were employed

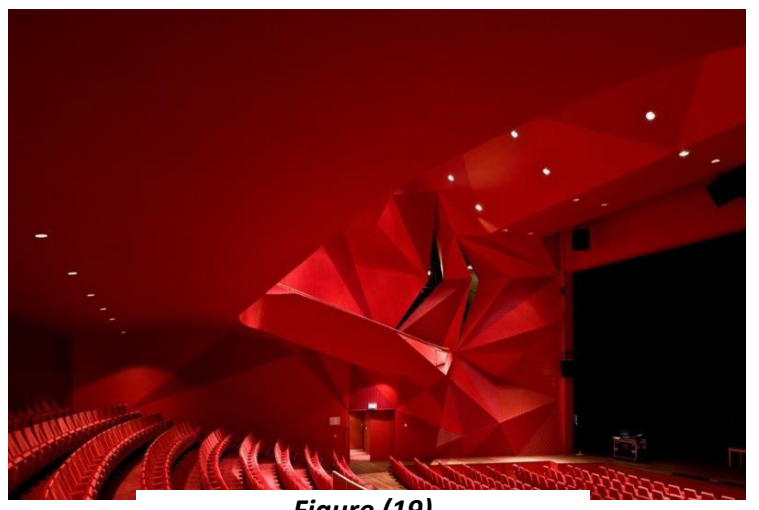

Figure (19)

in a distinct and varied manner that added a breathtaking beauty with the help of lighting distributed artistically 
that reflected a work of art in

the design. In addition, modern

furniture was used matching

the colors of the walls and the

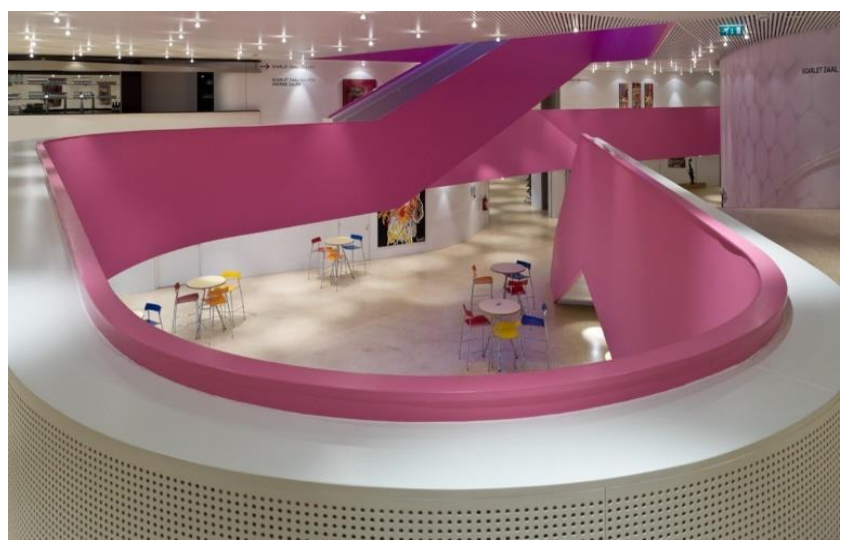

Figure (20)

edges of the stairs that have been varied in their direction and design, as well as placing artistic paintings on the walls that have added its aesthetic touch to the building. As for the walls and ceilings, they haddiverse designs with interlocking sets of geometric shapes with variable heights, on which lines were drawn that increased the aesthetic design.We can note how the theater hall emerged in red, increasing the mental focus and attention

that the designer wanted for the attendees to

interact with theatrical events in its full details,

and we find that all these formations gave the

building great artistic value and helped the

building in imposing its artistic identity,

surpassing the stagnancy of buildings' designs.

The combination of these internal and external

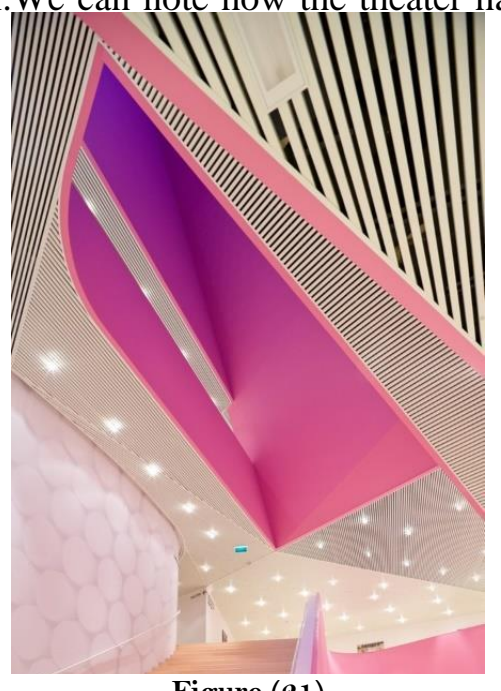

Figure (21)

optical formations highlighted the building's artistic identity and made it a center of attraction for tourists and visitors.

From the previous chapter, we can draw the following conclusions:

The optical formations of various kinds, including the aesthetic touch it adds and the expression of the various creative and unfamiliar ideas of the designer creates a radical change in the viewer's fixed rules and expectations. For the creative touch in overlap, connection, separation, penetration, segmentation of shapes and colors, and the choice of materials needed to implement these designs have the greatest role in highlighting landmarks of urban identity. 
Where we found that the optical formation in its various forms is the essential architecture cloak of which it boasts, along with its creative ideas that express the reality of urban identity.

\section{Recommendation:}

Study of the modern optical formations in terms of the materials used in their implementation, and did the development in the materials used increase the impact of highlighting the architectural identity?

\section{Sources and References}

[1] Al Monajed in contemporary Arabic, Dar Al-Mashriq, Beirut

[2] Lisan Al-Arab, on the website www.ar.wikisource.org

[3] AfifBahnassi, on the website of the Arabic Encyclopedia www.arab encyclopeda.com

[4] Al Saeed, Shaker Hassan, Ana Al NoqtaFawqaFaa Al Harf, the House of General Cultural Affairs Baghdad, 1998

[5] SamaherBint Abdul Rahman Falatah, The Art of Visual Delusion and the Possibility of Creating New Designs for Mineralogy Jewelry, King Saud University, Saudi Arabia, 2008

[6] Dr. Anayat Yusef Rafla, The Art of Visual Deception, Al-Taawun Foundation for Printing and Publishing, Cairo, 1975

[7] Ibrahim Jawad Kazem Al-Yusef Al-Husseini, Introduction to Reading Architecture, Baghdad, 2017

[8] Sherine Ihsan Sherzad, Principles in Art and Architecture, The Arab House, Year: None

[9] Nicholas Wade, optical illusions, translated by: Mai Al-Mudhafar, Dar Al-Mamoun for Translation and Publishing, Ministry of Culture and Media, Baghdad, 1988

[10] Anna Jaglarz, Perception and illusion in interior design, Department of Architecture Wroclaw University of Technology, St. George. Prusa 53/55, 50-317 Wroclaw, Poland.

[11] Anna Jaglarz, The Application of Optical Illusions in Interior Design in order to improve the Visual Size and Proportions of the Rooms, 2012Guzowski, Mary, Daylighting Sustainable Design, McGraw-Hill Companies, 2000. 